\title{
A SIMPLE CONTROL STRATEGY TO INCREASE THE TOTAL EFFICIENCY OF MULTI-CONVERTER SYSTEMS
}

\author{
Fabrício Hoff Dupont ${ }^{1}$, Jordi Zaragoza ${ }^{2}$, Cassiano Rech $^{1}$, José Renes Pinheiro ${ }^{1}$ \\ ${ }^{1}$ Power Electronics and Control Research Group (GEPOC) — Federal University of Santa Maria (UFSM) \\ Av. Roraima, 1000, 97105-900, Santa Maria, Rio Grande do Sul, Brazil \\ ${ }^{2}$ Terrassa Industrial Electronics Group (TIEG) — Technical University of Catalonia (UPC) \\ C. Colom, 1, 08222, Terrassa, Barcelona, Spain \\ Contact: fhd@ieee.org
}

\begin{abstract}
This paper presents a simple control strategy that is employed together with an optimization methodology to achieve optimal efficiency in systems composed by multiple parallel converters. An external loop is responsible for regulating the dc bus voltage and providing a current reference that is weighted in function of the power processed by the system, and then generating optimal current references for each converter. This enable the system to operate with optimal efficiency for all its load range. Experimental results demonstrate the superior performance of the proposed strategy, improving the efficiency of the system in almost $10 \%$ under light load operation in comparison with the conventional strategy of equal power sharing among converters.
\end{abstract}

Keywords-Parallel converters, digital control, efficiency maximization, supervisory control, active current sharing, photovoltaic systems

\section{INTRODUCTION}

Renewable energy sources are becoming more popular each day. However, its cost is still high and its efficiency may still be considered low. Thus, improving the efficiency and a better use the installed power generation capacity are fundamental objectives for the design of systems fed by renewable energy sources. Photovoltaic systems, for example, are formed by PV modules and power conditioning stages. The improvement of the efficiency of PV cells mainly depends on technological aspects and is usually associated with a significant increase in costs. On the other hand, recent maximum power point tracking (MPPT) algorithms achieve efficiencies higher than $99 \%$ [1]. Therefore, the power conversion stage is the one that allows better efficiency improvements for these type of systems.

It is shown in [2] that for the Brazilian territory the largest share of the energy processed by PV modules lies in the range of $20 \%$ to $60 \%$ of the peak installed power. For this reason it is essential that the converters in charge of the interface between the PV modules and the loads can operate with high efficiency under light load conditions.

One strategy that enable the efficiency improvement is the use parallel connected converters. With lower power ratings, these converters can be assembled employing devices with reduced losses, besides enabling the modularity of installations. In this kind of application, the droop method is one of the control strategies that is most employed, mainly in microgrids, since it is easy adapt for converters with different power ratings [3], [4]. However, the objective of this method is just to share the processed power in a balanced way. Aiming to improve the overall efficiency of parallel converters some strategies has been recently proposed, as the connection or disconnection of phases in interleaved converters as a function of the load demand [5], the perturbation and observation of power distribution between converters [6], or the employment of digital filters for a passive current sharing [7]. Although these strategies does not ensure that the system will operate with optimal efficiency in all its load range.

This paper presents a simple control strategy that, employed together with the optimization methodology proposed by [8], allows a multi-converter system to operate with optimal efficiency in all its operating points. In turn, experimental results shows that just with the presented control strategy the efficiency of a system of parallel converters is significantly improved for light load operation.

\section{TOTAL SyStem EFFICIENCY Optimization}

The efficiency of a static converter is defined by the well known equation

$$
\eta=\frac{p_{\text {out }}}{p_{\text {in }}}
$$

being $p_{\text {in }}$ the power drained from the source and $p_{\text {out }}$ the power delivered to the load. There are many factors that introduces losses in the power conversion, including technological and constructive aspects. Besides, the operating point of the converter also impacts in the conversion losses. Therefore, the efficiency of converters is commonly presented in the form of curves obtained experimentally or from theoretical analysis. Most of these curves can be approximated by the second order function defined by

$$
\eta\left(p_{\text {in }}\right)=\frac{\alpha_{1} p_{\text {in }}+\alpha_{0}}{p_{\text {in }}^{2}+\beta_{1} p_{\text {in }}+\beta_{0}}
$$

being $\alpha_{1}, \alpha_{0}, \beta_{1}$ and $\beta_{0}$ coefficients that can be obtained with the aid of a curve fitting algorithm.

In systems formed by $n_{c}$ parallel converters, the total system efficiency is also evaluated by the relationship between the output power and the sum of the input power. Once the operating point of the converters directly affect its 
efficiency, it is reasonable to suppose that for the same output power, different power distributions will result in different total system efficiencies. Thus, a highly desirable feature of a multi-converter system is to ensure its operation under a condition of optimal efficiency for all possible load range. Knowing that $p_{\text {out }}=p_{\text {in }} \eta\left(p_{\text {in }}\right)$, one can rewrite (1) for $n_{c}$ parallel converters as an optimization problem as

$$
\begin{aligned}
\eta\left(p_{1}, \ldots, p_{\text {in }, n_{\mathrm{c}}}\right)_{\operatorname{máx}} & = \\
\min _{p_{\text {in }}} & \left(-\frac{\sum_{m=1}^{n_{\mathrm{c}}} \frac{p_{\mathrm{in}, m}\left(\alpha_{1, m} p_{\mathrm{in}, m}+\alpha_{0, m}\right)}{p_{\mathrm{in}, m}^{2}+\beta_{1, m} p_{\mathrm{in}, m}+\beta_{0, m}}}{\sum_{m=1}^{n_{\mathrm{c}}} p_{\mathrm{in}, m}}\right)
\end{aligned}
$$

whose solution allows one to obtain a set of optimum values for $p_{\text {in, } m}$, ensuring maximum system efficiency for all its possible operating points.

As highlighted in [8] the optimization problem defined in (3) is nonlinear, constrained, and may feature multiple global minima. The first constraint implies that the sum of the powers processed by each converters must be equal to the desired output power, which defines a linear equality. A linear inequality also defines that the power processed by each converter must be smaller than its maximum power. Moreover, the search interval must be bounded to the power range that each converter is able to handle. To solve this problem a four stage methodology has been proposed, which is briefly summarized below.

\section{A. Initialization}

In the initialization stage the characteristics of the system, as the number of converters, its power ratings and the coefficients $\alpha_{1}, \alpha_{0}, \beta_{1}$ and $\beta_{0}$ of the efficiency curves of each converter are defined. From these information, the optimization problem is set up and executed for all the operating points to be optimized.

\section{B. Global optimization}

Due to the complexity of the problem, most of the traditional optimization algorithms may stuck in a local minimum and does not find the point of maximum system efficiency. To overcome this problem, a genetic algorithm (GA) is employed in a stage of global optimization. The GA are search and optimization methods based on the natural selection principle and is one of the evolutionary computation techniques most used nowadays [9], [10]. It is worth to notice that the GA is a robust method that does not need information about the derivatives of the objective function and performs the search throughout several points of the solution hyperplane simultaneously.

Since the GA is a stochastic algorithm, the results may not have the required precision. For this reason, the GA is used to quickly determine the region in which the optimal solution lies. Without waiting the GA to find the optimal solution with full precision, the algorithm is terminated and the best chromosome is employed as an initial guess for a local optimizer algorithm.

\section{Local optimization}

Traditional optimization methods have been extensively developed to quickly find the solution of well defined convex functions. Once GA provided a good guess of the optimal solution, a local optimizer is employed to refine results and obtain the optimal power distribution between converters that results in the maximum system efficiency.

The local optimizer employed in the methodology solves the Karush-Kuhn-Tucker (KKT) equations, which are necessary conditions to the optimization of constrained problems [11]. Specifically, a nonlinear optimization algorithm based on the sequential quadratic programming (SQP) is used. These algorithms perform an inline search using a figure of merit similar to the proposed in [12], [13] and approximates the Newton method for constrained optimization problems, as detailed in [14].

\section{Ambiguity resolution}

In systems with multiple equal converters, multiple global optimum points are verified. This means that different power distributions among converters can provide the maximum system efficiency. Again, due to the stochastic characteristic of the GA, one can not guarantee that the optimal solution found for a given operating point is correlated with the optimal solutions nearby. Thus, a stage of ambiguity resolution is employed to provide a logical power distribution among converters, avoiding unnecessary redistributions to provide the same total system efficiency.

\section{Control Strategy}

More than obtain the power distribution that provides the optimal total efficiency, it is also important to design an adequate control strategy that enable the system to operate in accordance to the references established by the optimization methodology. In this sense, the main objective of this paper is to present a simple and effective choice to this task, providing an adequate dynamic response and optimal efficiency for all operating points.

The proposed control strategy is based on the active current sharing among converters. An external loop has the objective of dc bus voltage $\left(v_{\mathrm{o}}\right)$ regulation, generating an input current reference $i_{\mathrm{r}}$ for the system that is send to the supervisor block. From a decision variable $\delta_{v}$ that represents the power consumed by the load, the supervisor applies a convex weighting in the current reference. These weighted references $i_{\mathrm{r}, 1}, i_{\mathrm{r}, 2}$ and $i_{\mathrm{r}, 3}$ are then applied to internal control loops which are in charge of input current regulation for each converter. Thus ensuring that each one processes the optimal power share established by the optimization methodology. A block diagram of the proposed strategy is depicted by Fig. 1.

Three boost converters are used to demonstrate the control strategy investigated in this paper, whose specifications are given by Table I. Employing the digital power meter Yokogawa WT1600 some efficiency samples were acquired, which are depicted by Fig. 2. As it can be observed, the efficiency values are almost the same for all converters in each power level evaluated. Thus, it is assumed that the three converters can be represented by the same efficiency 


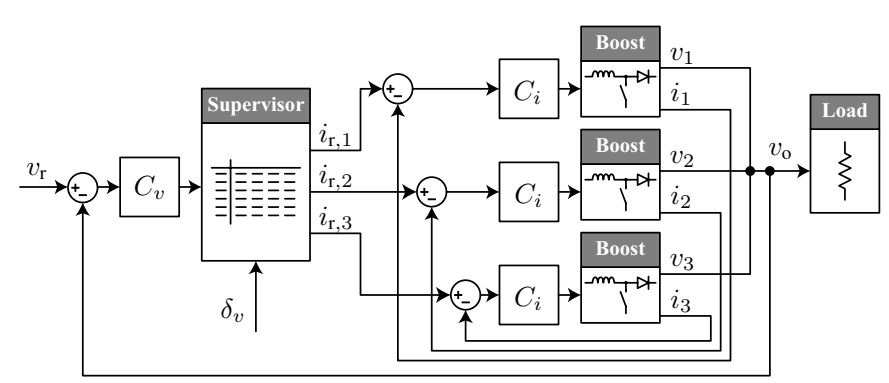

Figure 1: Control strategy employed to maximize the total efficiency of a multi-converter system.

Table I: Parameters of the experimental prototype.

\begin{tabular}{cccc}
\hline Parameter & Value & Parameter & Value \\
\hline$V_{i}$ & $150 \mathrm{~V}$ & $V_{o}$ & $400 \mathrm{~V}$ \\
$P_{o}$ & $1200 \mathrm{~W}$ & $P_{o, 1}, P_{o, 2}, P_{o, 3}$ & $400 \mathrm{~W}$ \\
$L_{\mathrm{B}}$ & $6 \mathrm{mH}$ & $F_{s}$ & $10 \mathrm{kHz}$ \\
$C_{\mathrm{o}}$ & $680 \mu \mathrm{F}$ & $D$ & 0.625 \\
\hline
\end{tabular}

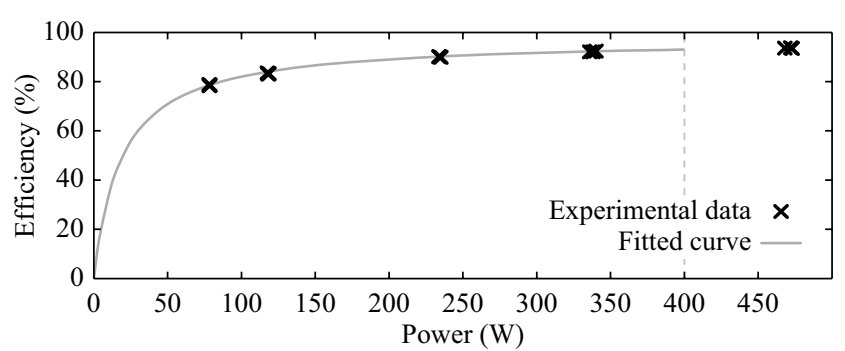

Figure 2: Experimental efficiency values and efficiency curve employed in the optimization methodology.

curve. Applying the experimental samples in a curve fitting algorithm the coefficients

$$
\left\{\begin{array}{l}
a_{1}=2498.5 \\
a_{0}=0.0352 \\
b_{1}=25648.4 \\
b_{0}=1204.1
\end{array}\right.
$$

of (2) are obtained, which in turn provides the curve also shown in Fig. 2.

Applying the optimization methodology for this case, the optimal power distributions depicted by Fig. 3(a) are obtained for the entire load range. Normalizing these curves in function of the power demand for each operating point, one has the weighting curves that must be applied to the current reference $i_{\mathrm{r}}$ in order to obtain the references $i_{\mathrm{r}, 1 \ldots 3}$ to be applied to each converter and achieve the expected power distribution. These weighting curves are illustrated by Fig. 3(b).

As demonstrated in [15], [16] the small signal dynamic model of the boost converter is given by the second order

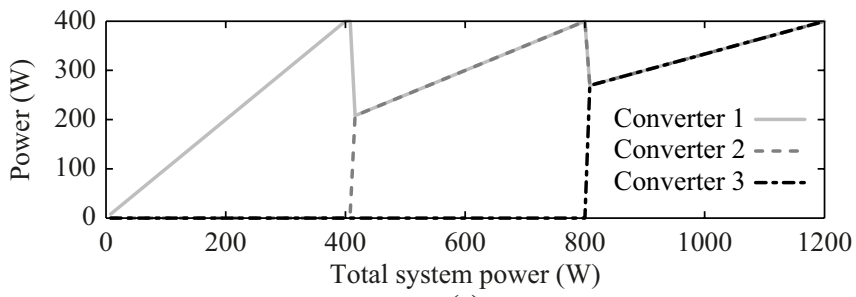

(a)

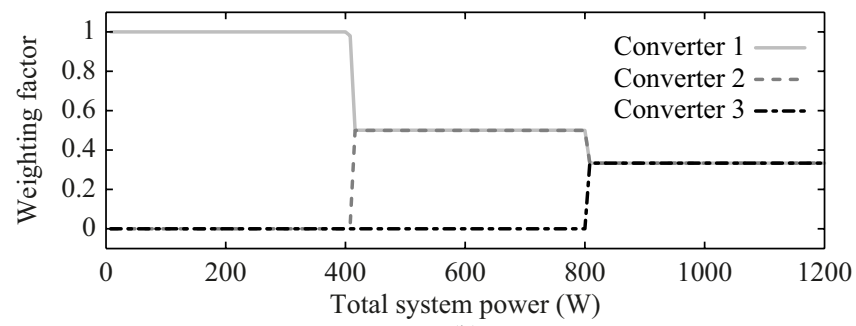

(b)

Figure 3: Results obtained with the efficiency optimization methodology (a) optimal power distribution; (b) weighting values to be assigned to the current reference $i_{\mathrm{r}}$.

transfer function

$$
G(s)=G_{\mathrm{dc}} \frac{\left(\frac{s}{\omega_{z}}+1\right)}{\left(\frac{s}{\omega_{0}}\right)^{2}+\frac{s}{Q \omega_{0}}+1}
$$

being $G_{\mathrm{dc}}$ the dc gain, $\omega_{z}$ the frequency of the zero, $\omega_{0}$ the natural frequency of the plant and $Q$ the quality factor. For the transfer function of the input current $i$ by the control signal $d$ for each converter, these parameters are obtained by means of

$$
\begin{array}{r}
G_{\mathrm{dc}, i}=\frac{2 V_{i}}{R_{L} D^{\prime 3}} \quad Q=D^{\prime} R_{L} \sqrt{\frac{C_{\mathrm{o}}}{L_{\mathrm{B}}}} \\
\omega_{z}=\frac{2}{C_{\mathrm{o}} R_{L}} \quad \omega_{0}=\frac{D^{\prime}}{\sqrt{L_{\mathrm{B}} C_{\mathrm{o}}}}
\end{array}
$$

where $D^{\prime}=1-D$ and $R_{L}$ is the load resistance for the nominal power of each converter $\left(R_{L}=400 \Omega\right)$.

In discrete control systems for power electronics the transport delay involving the sampling of variables and the update of the PWM modulator should be considered [17]. Thus, applying the specifications of Table I in (6), substituting in (5), discretizing by a zero-order-hold $(\mathrm{ZOH})$ with the same frequency of $F_{s}$ and including a delay of one sample, one has

$$
G_{i}(z)=\frac{6.668 z-6.663}{z^{3}-1.999 z^{2}+z} .
$$

Assuming that the current loops are much faster than the voltage control loop, the output stage of the system can be simplified by an impedance fed by a current source. This impedance is formed by load resistances and the bus capacitor. Indeed, in steady state the load current is given by $i_{\text {out }}=i_{\text {in }} D^{\prime}$. This leads to the output voltage by the input current transfer function, which is given by

$$
G_{v}(s)=G_{\mathrm{dc}, v} \frac{1}{s+\omega_{p}}
$$


being

$$
G_{\mathrm{dc}, v}=\frac{1-D}{C_{\mathrm{o}}} \quad \omega_{p}=\frac{1}{R_{\mathrm{sys}} C_{\mathrm{o}}}
$$

where $R_{\text {sys }}$ is the load resistance for the nominal power of the system $\left(R_{\text {sys }}=133.3 \Omega\right)$.

Applying the specifications of the converters in (9) and discretizing $(8)$ by a $\mathrm{ZOH}$ with $F_{s}$, one has

$$
G_{v}(z)=\frac{0.05512}{z-0.9989}
$$

To design the current controllers it has been defined that its bandwidth should be less than one decade below the switching frequency and with a phase margin greater than $45^{\circ}$. From this criteria a PI controller has been designed, whose transfer function is given by

$$
C_{i}(z)=\frac{48.795 \times 10^{-3} z-44.411 \times 10^{-3}}{z-1}
$$

which provide a bandwidth of $515 \mathrm{~Hz}$ and a phase margin of $47.3^{\circ}$.

The design of the voltage controller has the criteria of a bandwidth less than one decade below the current loop and a phase margin greater than $60^{\circ}$. In this case, a PI controller with low-pass filter has been designed, which transfer function is given by

$$
C_{v}(z)=\frac{13.97 \times 10^{-3} z-13.954 \times 10^{-3}}{z^{2}-1.952 z+952.15 \times 10^{-3}}
$$

that provide a phase margin of $71^{\circ}$ and a bandwidth of $24.4 \mathrm{~Hz}$.

The next step in the control system design is the choice of a decision variable for the power sharing strategy. For the system under analysis, two variables directly represents the power drained by the load: the total input current and the output current. Under the implementation point of view, the use of the total input current usually has lass impact over the system costs, , since that input current sensors are usually available in the case of boost converters, while the use of the output current requires an additional sensor.

If on one hand the output current reflects the instantaneously drained energy, even during transient periods, on the other hand, the total input current represents the energy consumption only in steady-state. During transients, energy variations that are necessary to lead the converters to different operating points can result in inadequate power redistributions. This affects the transient response of the system as a whole and may even cause its instability. One way to overcome this problem is to use a filter with a very low bandwidth, such that the supervisory control responds only based on steady-state values. However, this should be done carefully, as large power steps could make the system unable to supply the load demand or force active converters to work under serious overload conditions.

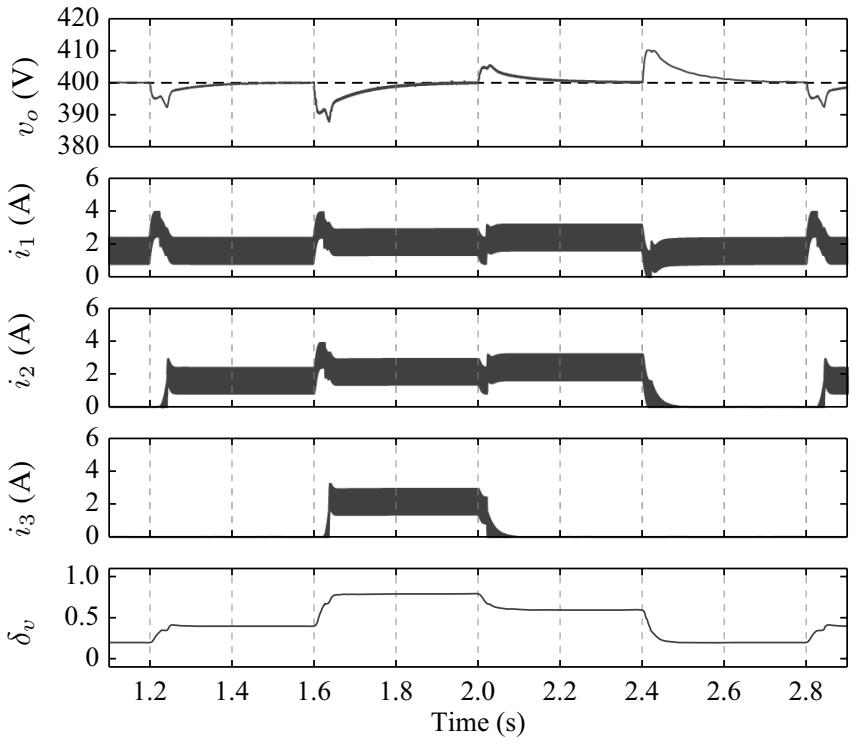

Figure 4: Simulation results employing the total input current as decision variable for the power sharing supervisor.

\section{Simulation Results}

To investigate the overall dynamic behavior of the system when employing the total input current or the output current as decision variables, this section presents simulation results carried out with Simulink. The simulation model follows the block diagram of Fig. 1, while the supervisor block is implemented by means of lookup-tables that stores the weighting curves shown at Fig. 3(b) for each converter.

As previously highlighted, when using the input current as decision variable it is desirable to use a low-pass filter so that the supervisory controller responds only over near steady-state values. For this simulation a first order lowpass filter with cutoff frequency of $10 \mathrm{~Hz}$ has been used. During the simulation a sequence of load steps are applied to evaluate the dynamic response of the controllers. The system is started with $20 \%$ of its maximum power, and after it is changed to $40 \%, 80 \%, 60 \%$ and back to $20 \%$. Results of this simulation are presented by Fig. 4, which shows the output voltage, the input currents of the three converters, and the normalized value of the decision variable. As observed, the reduced bandwidth of the decision variable damage the dynamic response of the output voltage, which is a negative point of this approach.

On the other side, employing the output current as decision variable enable better dynamic responses, as can be observed in the simulation results depicted by Fig. 5. Again, the same load sequence has been used. Differently from the input current, the output current may be directly used as a decision variable without additional filtering. Consequently, an improved dynamic response is observed for the output voltage. Thus, if a superior performance is a concern for the output voltage, the output current is the signal that enable better responses and it will be used in the experimental results presented in the next section. 

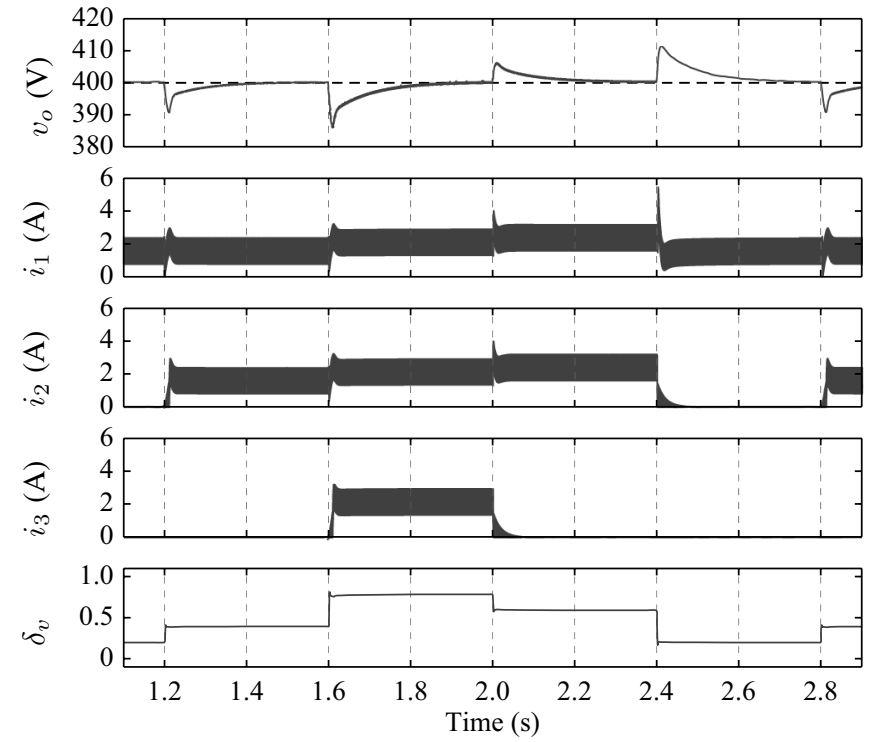

Figure 5: Simulation results employing the output current as decision variable for the power sharing supervisor.

\section{EXPERIMENTAL RESULTS}

The experimental validation of the control strategy presented in this paper is carried out with the aid of the hardware-in-the-loop (HIL) platform dSPACE DS1 103 which works together with Simulink to run a real time simulation of the system. This platform is composed by a PowerPC604e processor and a slave DSP TMS320F240 for advanced I/O purposes as the generation of the PWM signals.

Two current sharing strategies are experimentally compared. In the first test the processed power is shared equally among each converter, as conventionally done in most control strategies for parallel converters. In the second case the control strategy present so far is evaluated employing the output current as decision variable.

Load steps are applied each $400 \mathrm{~ms}$ to evaluate the dynamic response of each strategy. This steps follows the sequence: $80 \%, 60 \%, 40 \%$, and $60 \%$ of the maximum power of the system. In experimental tests the load has not been reduced to $20 \%$ since the converters would operate in discontinuous conduction mode (DCM) for the strategy of equal power sharing, and this mode is not supported by the controllers. On the other hand, the restriction imposed by the DCM is significantly alleviated for the proposed control approach, since only one converter is active under light load operation. This feature of the proposed scheme also enable the system to operate over a larger load range while ensuring the optimal conversion efficiency.

Experimental results for the strategy of equal power sharing are shown by Fig. 6. This strategy presents an adequate dynamic response for the output voltage. The maximum overshoot verified during load changes is of $1.4 \%$, observed when the load changes from $60 \%$ to $80 \%$ of the maximum system power. On the other hand, the equal power sharing has the disadvantage of poorer efficiency for light

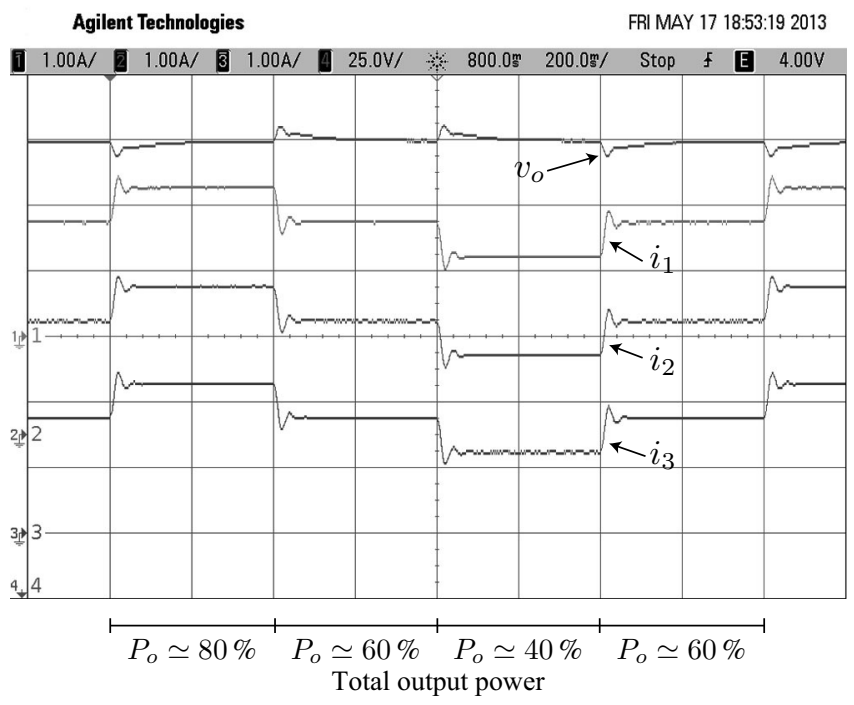

Figure 6: Experimental results for load switching employing equal power distribution for all converters.

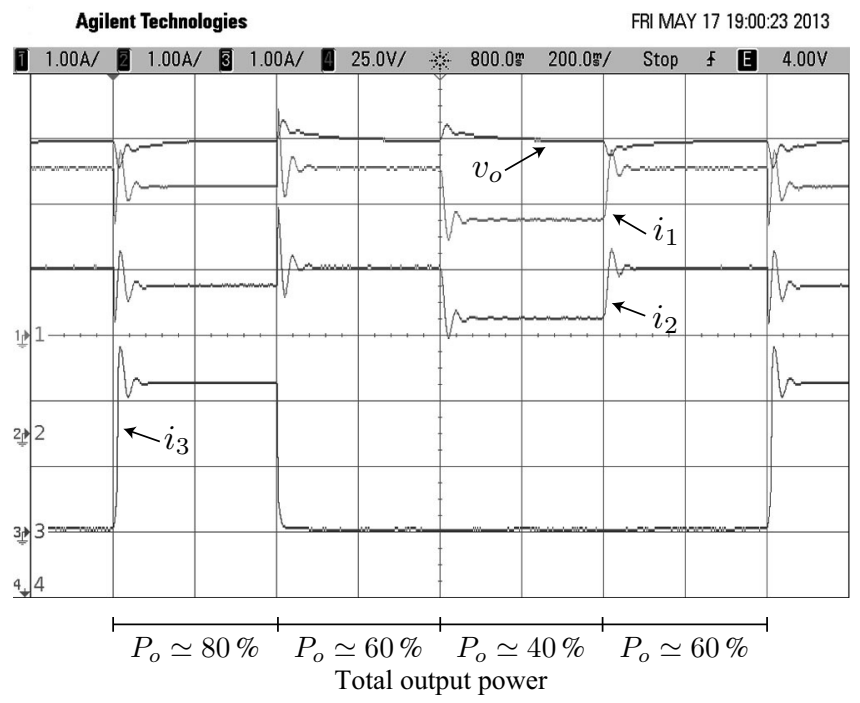

Figure 7: Experimental results for load switching employing the proposed sharing scheme.

load conditions and, in this case, also requiring controllers that support the DCM.

Fig. 7 depicts the experimental results for the control strategy presented in this paper. It can be easily seen that the Converter 3 is employed only when the load demand is of $80 \%$ of the maximum system efficiency. For $60 \%$ and $40 \%$ of power the load is fed only by converters 1 and 2, as defined by the curves presented by Fig. 3. Even featuring slightly higher overshoots, the output voltage also has an adequate dynamic response. The maximum overshoot is of $2.3 \%$ during the transient from $60 \%$ to $80 \%$ of the maximum system power. This minor increase on the maximum overshoot is mainly due to the dynamics involved in the power redistribution between converters.

A comparison between the efficiencies achieved with the 


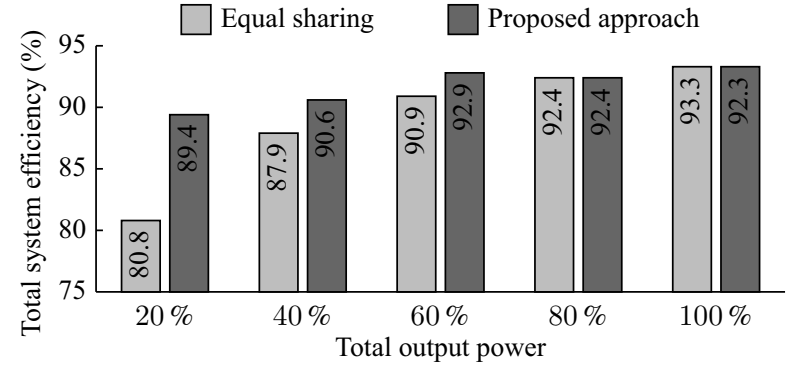

Figure 8: Comparison of experimental efficiencies between equal power sharing and the proposed control strategy.

employment of each strategy is shown by Fig. 8. It must be highlighted that the control approach presented in this paper, together with the optimization methodology proposed in [8], allows an improvement o amost $10 \%$ on the system efficiency when the it operates at $20 \%$ of power. From theoretical efficiency curves of converters, one has that the euro efficiency for the strategy of equal power sharing is of $85.52 \%$, while the control strategy presented in this paper reaches $90.96 \%$, without any hardware modification.

\section{CONCLUSIONS}

This paper presented a control strategy that enable the total efficiency improvement for multi-converter systems. The presented strategy employs the optimal power distribution curves obtained by means of an optimization methodology and implements a simple supervisory control that weights the current reference generated by a voltage controller. Simulation results show that the output current is a better option to be employed as decision variable for the supervisory control, as it represents the load power demand instantaneously. Moreover, experimental results shows that the presented strategy almost do not interfere in the output voltage behavior. On the hand, the presented approach enable an efficiency improvement of almost $10 \%$ for operation under light load demands.

\section{ACKNOWLEDGEMENT}

This work is supported by CNPq (National Council of Scientific and Technological Development), CAPES (Brazilian Commission for Higher Education), FAPERGS (Foundation for Supporting Research from the State of Rio Grande do Sul), and RURALGRID project on CTP frame with support of the Comissionant per a Universitats i Recerca del Departament d'Innovació, Universitats i Empresa of the Generalitat de Catalunya.

\section{REFERENCES}

[1] T. Esram and P. L. Chapman. Comparison of photovoltaic array maximum power point tracking techniques. IEEE Trans. Energy Convers., 22(2):439449, 2007.

[2] F. H. Dupont, C. Rech, and J. R. Pinheiro. A methodology to obtain the equations for the calculation of the weighted average efficiency applied to photovoltaic systems. In 10th IEEE/IAS Int. Conf. on Ind. Appl., pages 1-8, 2012.
[3] K. Kurohane, T. Senjyu, A. Yona, N. Urasaki, T. Goya, and T. Funabashi. A hybrid smart AC/DC power system. IEEE Trans. on Smart Grid, 1(2):199-204, 2010.

[4] R. Majumder, B. Chaudhuri, A. Ghosh, G. Ledwich, and F. Zare. Improvement of stability and load sharing in an autonomous microgrid using supplementary droop control loop. IEEE Trans. on Power Syst., 25(2):796808, 2010.

[5] P. Zumel, C. Fernnndez, A. de Castro, and O. Garcia. Efficiency improvement in multiphase converter by changing dynamically the number of phases. In $37 \mathrm{th}$ IEEE Power Electron. Spec. Conf., pages 1-6, 2006.

[6] J. A. Abu-Qahouq, L. Huang, D. Huard, and A. Hallberg. Novel current sharing schemes for multiphase converters with digital controller implementation. In 22nd Annual IEEE Applied Power Electron. Conf., pages 148-156, 2007.

[7] A. Kelly. Current share in multiphase DC-DC converters using digital filtering techniques. IEEE Trans. on Power Electron., 24(1):212-220, 2009.

[8] F. H. Dupont, J. Zaragoza, C. Rech, and J. R. Pinheiro. A new method to improve the total efficiency of parallel converters. Submited to the 12th Brazilian Power Electronics Conference. In Brazilian Power Electron. Conf. (COBEP), 2013.

[9] Randy L. Haupt and Douglas H. Werner. Genetic algorithms in electromagnetics. IEEE Press, Hoboken, NJ, 2007.

[10] M. H. Ershadi, M. B. Poudeh, and S. Eshtehardiha. Fuzzy logic controller based genetic algorithm on the step-down converter. In Int. Conf. on Smart Manuf. Appl., pages 324-328, 2008.

[11] Hsien-Chung Wu. The Karush-Kuhn-Tucker optimality conditions in an optimization problem with intervalvalued objective function. Eur. J. of Operational Research, 176(1):46-59, 2007.

[12] S. P. Han. A globally convergent method for nonlinear programming. J. of Optim. Theory and Appl., 22(3):297-309, 1977.

[13] M. Powell. A fast algorithm for nonlinearly constrained optimization calculations. In G. Watson, editor, Numerical Analysis, volume 630 of Lecture Notes in Mathematics, pages 144-157. Springer, Berlin, 1978.

[14] R. Fletcher. Practical methods of optimization. Wiley, Chichester, 2nd edition, 1987.

[15] Robert W. Erickson and Dragan Maksimovic. Fundamentals of power electronics. Kluwer Academic, Norwell, 2nd edition, 2001.

[16] George C. Verghese. Dynamic modeling and control in power electronics. In W. S. Levine, editor, The control handbook, pages 1413-1423. IEEE Press, Boca Raton, $\mathrm{Fl}, 1996$.

[17] Simone Buso and P. Mattavelli. Digital control in power electronics. Morgan \& Claypool, San Rafael, 2006. 